\title{
(2) \\ Postmarketing studies: can they provide a safety net for COVID-19 vaccines in the UK?
} OPEN ACCESS

\section{Sandeep Dhanda (1) ,1,2 Vicki Osborne (1) ,1,2 Elizabeth Lynn (1) , ${ }^{1,2}$ Saad Shakir (1) ${ }^{1,2}$}

\subsection{6/bmjebm-2020-111507}

${ }^{1}$ Drug Safety Research Unit,

Southampton, UK

${ }^{2}$ School of Pharmacy and

Biomedical Sciences, University of Portsmouth, Portsmouth, UK

Correspondence to: Dr Sandeep Dhanda, Drug Safety Research Unit, Southampton S031 1AA, UK; sandeep.dhanda@dsru.org
(A) Check for updates

(- Author(s) (or their employer(s)) 2022. Re-use permitted under CC BY-NC. No commercial re-use. See rights and permissions. Published by BMJ.

To cite: Dhanda S, Osborne V, Lynn E, et al. BMJ Evidence-Based Medicine 2022;27:1-6.

\section{Abstract}

In the current era of the COVID-19 pandemic, the world has never been more interested in the process of vaccine development. While researchers across the globe race to find an effective yet safe vaccine to protect populations from the newly emergent SARS-CoV-2 virus, more than one-third of the world has been subjected to either full or partial lockdown measures. With communities having felt the burden of prolonged isolation, finding a safe and efficacious vaccine will yield direct beneficial effects on protecting against COVID-19 morbidity and mortality and help relieve the psychological and economic load on communities living with COVID-19. There is hope that with the extraordinary efforts of scientists a vaccine will become available. However, given the global public health crisis, development of a COVID-19 vaccine will need to be fast tracked through the usual prelicensing development stages and introduced with limited clinical trial data compared with those vaccines that are developed conventionally over more than a decade. In this scenario, surveillance of the vaccine in the real world becomes even more paramount. This responsibility falls to observational researchers who can provide an essential safety net by continuing to monitor the effectiveness and safety of a COVID-19 vaccine after licensing. Postauthorisation observational studies for safety and effectiveness are complementary to prelaunch clinical trials and not a replacement. In this paper, we highlight the importance of postmarketing studies for future newly licensed COVID-19 vaccines and the key epidemiological considerations.

\section{Vaccine development considerations for COVID-19}

The normal process for developing a vaccine is broadly similar to drug development. It is lengthy and can take $10-15$ years. ${ }^{1}$ Animal studies follow chemical and biological development researching the immune response; the vaccine is then tested in humans in phases I-III clinical trials. ${ }^{2}$ Assessing the efficacy of a vaccine in phase III studies takes longer than medicines because the outcomes are not simply the beneficial effects of a drug on a disease but a combination of examining effects on biomarkers, for example, antibodies, and the reduction in the incidence of developing the disease compared with people who were not vaccinated. Furthermore, as vaccines are given to large numbers of healthy individuals over a short period of time, testing in larger cohorts is required with often a greater emphasis on detection of adverse events than with medicines. ${ }^{3}$

The usual process of vaccine development has been considered too slow and impractical in pandemics such as COVID-19. ${ }^{4}$ Initial modelling by the Imperial College COVID-19 Response Team, given an estimated $R_{0}$ of 2.4 (in the scenario of an absence of any control measures or individual behaviour changes), predicted infection in $81 \%$ of Great Britain (GB) and US population, with an estimated 510000 deaths in GB and 2.2 million in the USA. ${ }^{5}$ However, recent preprint evidence suggests lower infection fatality rate (IFR) than speculated in the early days of the pandemic; a review of population-based seroprevalence studies suggest a median IFR of $0.24 \% .^{6}$ Where in an ideal world vaccine development would involve several years of rigorous clinical trials, in reflection of a potentially highly contagious virus pandemic with predicted high mortality and serious morbidity, the process has been prepared to be dramatically shortened. With the uncertainty surrounding COVID-19, the hope is to develop, test and release a vaccine within 12-18 months. ${ }^{2}$

Accelerated vaccine development for COVID-19 includes the evaluation of novel vaccine mechanisms; advances in genetics and biotechnology are contributing to this innovation, with the production of gene-based vaccines made from DNA or messenger RNA focusing on the spike protein (S-protein). ${ }^{7}$ To date, more than 176 vaccine candidates have been proposed globally with 34 in clinical evaluation and 142 in preclinical evaluation. ${ }^{8}$ Expedited advancement of these vaccines has resulted in animal studies and phase I human clinical trials occurring in parallel and progression to phase II trials before results of phase I trials evaluating adverse events are publicly released; additionally, fast-tracking through phase II and III clinical trials is also foreseen. ${ }^{29}$ However, a fast track phase II/III approval trial of 6 months or less could potentially miss changes in serum antibodies that occur after longer periods post immunisation; a MERS-CoVphase I trial vaccine trial showed a disappearance in spike-specific binding antibodies at month $6 .{ }^{10}$ Thus, considerable shortening of phase II and III randomised controlled trials in the development and testing of COVID-19 vaccines could potentially miss important results related to safety and efficacy. Furthermore, to 
Box 1 The path to COVID-19 vaccine licensing, linking premarketing and postmarketing activities

- The risk of advancing vaccines expeditiously through the premarketing schedule versus the benefit of having a vaccine available needs careful balancing.

- Fundamentally, it is important that the speed of vaccine development does not come at the cost of vaccine safety and effectiveness.

- For COVID-19 vaccines, with regulatory agencies engaging with vaccine developers much earlier than usual, an accelerated regulatory approval pathway is possible, even likely, whereby the vaccine may be licensed as soon as efficacy is proven but with conditions of extensive postmarketing surveillance. ${ }^{1726}$

detect events occurring at a low frequency (eg, <1/10 000), a phase III trial would likely need to enrol at least 60000 individuals, with half in the control arm. ${ }^{11}$ This will not be feasible for a COVID-19 vaccine, as trial cohort sizes will be smaller and period of observation shortened. Thus, it is anticipated that the release of a vaccine will be based on favourable effects on biomarkers and less premarketing clinical data compared with standard marketing authorisation applications for vaccines (box 1).

Nevertheless, the quality, efficacy and safety of COVID-19 vaccines fast-tracked through the premarketing stage must be equal to vaccines developed using the standard method. It is a regulatory requirement that the marketing authorisation process for all vaccines and medicines include a risk management plan (RMP), which addresses important potential risks, important identified risks and missing information that are identified at the time of granting marketing authorisation. ${ }^{12}$ If the RMP includes proposals for postauthorisation safety and/or efficacy studies (which is the case in nearly all new vaccines and medicines), these are required to be done with agreed milestones. The timing and frequency of interim reporting and the completion of the final study report depend on the impact on the benefit/risk balance of the issue being monitored. Early and frequent interim reporting is expected to be necessary for vaccines and medicines for COVID19. While RMPs are required for each individual product, where necessary a single postauthorisation study can include examining the safety and/or efficacy of more than one product, this may be necessary for COVID-19 vaccines if they will be administered during the same period.

\section{Postmarketing studies for COVID-19 vaccines}

Premarketing uncertainties can be addressed in postmarketing studies (predominantly observational). ${ }^{13}$ Firstly, from a public health perspective, it is equally relevant to study how efficiently a vaccine reduces the incidence of disease in natural conditions of use in the population (effectiveness) in addition to its efficacy tested in the restricted conditions of clinical trial settings, comparing vaccinated with non-vaccinated individuals. Furthermore, effectiveness is dependent on vaccine potency, the performance of vaccine immunisation programmes and other factors that may influence the disease risk itself, such as seasonality. It is not possible to evaluate these during expedited premarketing development, but careful consideration is required in the postmarketing phase, in particular with seasonal changes in viral circulation falsely attributing low rates of COVID-19 hospital admissions or deaths to vaccine effectiveness. For this reason, postmarketing studies need to be designed with such considerations in mind.

Secondly, with limited premarketing data, obtaining real-time notification on lack of efficacy and safety of the vaccine when used routinely in the population is pivotal in ensuring early detection of signals that may arise during vaccination programmes. This allows regulatory agencies and other stakeholders, where necessary, to respond quickly to ensure that the vaccine retains a favourable benefit-risk profile.

\section{Active versus passive surveillance methods}

While national passive surveillance (eg, spontaneous reporting systems of suspected adverse drug reaction) is commonly used to detect vaccine-related signals, it relies on the cooperation of healthcare professionals (HCPs) or patients to spontaneously report the occurrence of safety and/or effectiveness outcomes. For seasonal influenza in children, Fluenz Tetra vaccination programmes have been evaluated successfully using postmarketing studies with passive methodology (including enhanced passive surveillance) since 2015 , after an initial pilot study in 2014. ${ }^{14}$ However, for a COVID-19 vaccine, it would be implausible to rely solely on spontaneous reporting or other passive systems; instead an active surveillance method is considered superior to passive methods.

Active methods involve organised data collection (primary or secondary) at specific time points on all vaccinated individuals participating in a study, irrespective of whether they experienced an adverse event or subsequently developed the disease for which the vaccine was indicated. Drawing experience from previous pandemics can assist in the development of COVID-19 active surveillance methods. During the 2009-2010 global H1N1 influenza A (swine influenza) pandemic, active surveillance using a prospective cohort study monitored self-reported serious adverse events and pregnancy outcomes in patients offered swine influenza vaccination; no significant safety issues were identified. ${ }^{15}$ For a COVID-19 vaccine, advanced active surveillance is likely to provide the most scientifically robust method for identifying potential safety or effectiveness concerns with real-world use.

Figure 1 summarises a proposed example of active surveillance for COVID-19 vaccines in the UK; a prospective cohort study with the aim to monitor the uptake, safety and effectiveness of the vaccine by using both primary (from vaccinees) and secondary (from HCPs) sources of data. This organised collection of data, as opposed to relying on voluntary reporting, reduces the potential for under-reporting and bias. Furthermore, obtaining information from both vaccinees and HCPs allows for more comprehensive identification of outcomes and supports validation, thereby improving quality of reports.

\section{Study design considerations}

\section{Availability and use of unvaccinated comparator}

The more familiar vaccine effectiveness evaluation methods involve comparing disease incidence between vaccinated and unvaccinated groups (cohort studies) or comparing rates of vaccination between infected cases and uninfected controls (casecontrol studies). ${ }^{16}$ For pandemics such as COVID-19, where it is expected that vaccination coverage in a population will be high, traditional methods for evaluating vaccine effectiveness may not be suitable due to the lack of availability of an unvaccinated comparator group and the associated ethical considerations. If, as a result of a gradual tiering system for vaccination 
Figure 1. Example of study flow for a COVID-19 active surveillance post-marketing study in the UK

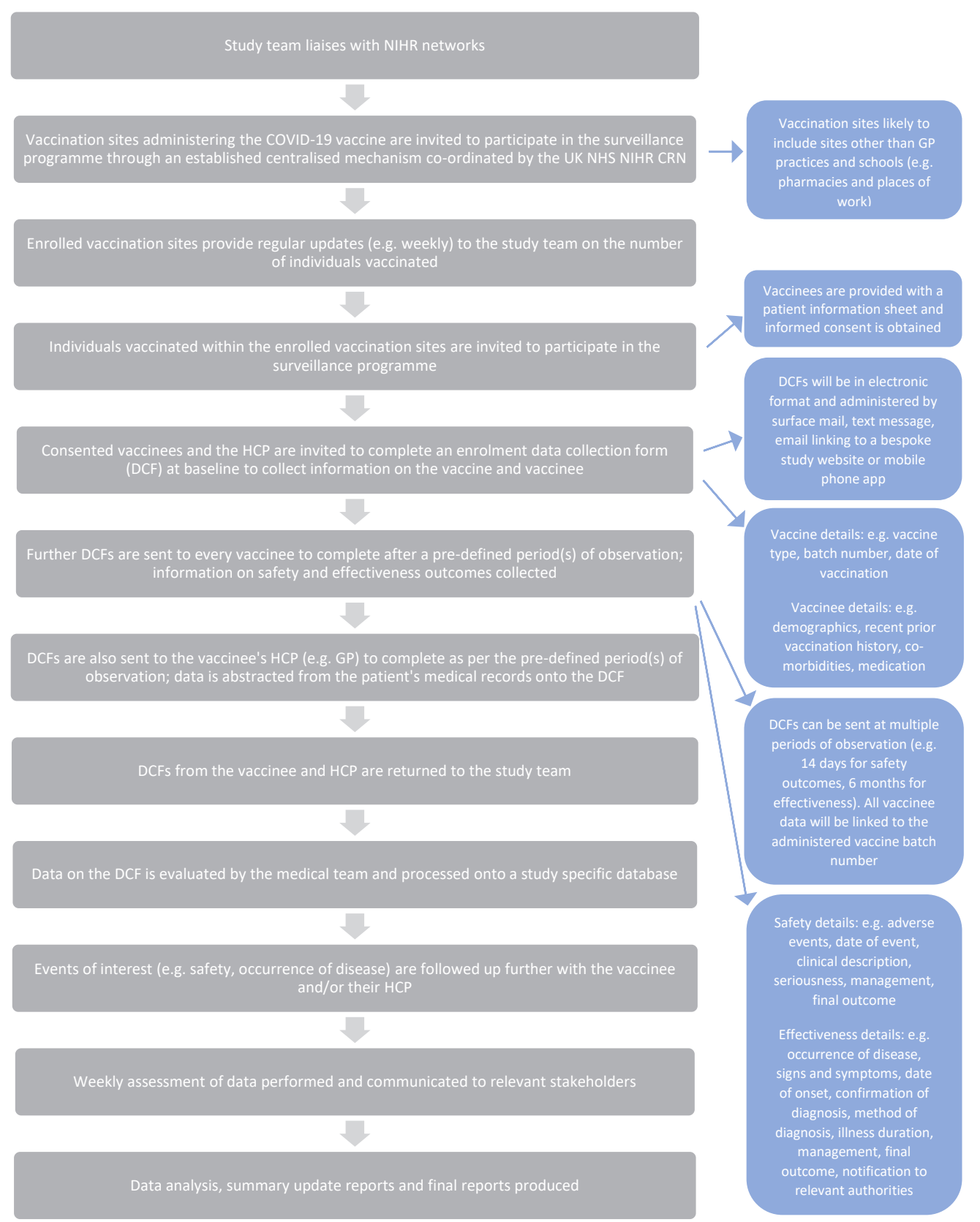

Figure 1 Example of study flow for a COVID-19 active surveillance postmarketing study in the UK. CRN, Clinical Research Networks; HCP, Healthcare professional; GP. General practitioner; NHS, National Health Service; NIHR, National Institute for Health Research.

whereby prioritisation is made for certain groups (eg, at-risk individuals, HCPs and older people) before reaching full vaccination coverage, an unvaccinated group is available, comparison of vaccinated groups to the unvaccinated population may not be appropriate due to different population characteristics with the potential for significant confounding and biases. Frequent sources of confounding include age, comorbidities, socioeconomic status and other factors that may influence the probability of being vaccinated or occurrence of certain adverse events postvaccination. Lastly, timeliness of results from comparative studies may be unacceptable for pandemics such as COVID-19, which demand urgent public health information.
In such scenarios, implementation of a faster and more responsive study design can address gaps in evidence in the immediate postmarketing period; an example of this is measuring disease incidence in vaccinees only. For a study on a COVID-19 vaccine for which there is a need to study both safety and effectiveness, in real time, a conventional cohort design of vaccinated individuals only may be appropriate (figure 1). Nonetheless, single-arm studies possess methodological challenges that require the same careful consideration in terms of statistical analyses as other study designs. Stratification by variables and powering the study adequately for subgroup analyses can provide further insight to potential differences in effectiveness and safety of the vaccine 
in certain populations, some of which may be at higher risk of disease complications and a fatal outcome. In addition, it is possible to include within cohort comparisons using nested casecontrol and self-controlled study designs to answer specific questions, for example, why do some vaccinees develop particular adverse events?

\section{Adverse event monitoring}

Rare or very rare serious adverse events have occurred after vaccination. ${ }^{17}$ For example, a vaccine-associated adverse event of interest is Guillain-Barre syndrome, for which the strongest causal link with a vaccine was observed during the 1976 US swine influenza programme; the associated risk of 1 case per 100000 resulted in suspension of the vaccination programme. ${ }^{18}$ More recently, a causal link was found between the $2009 \mathrm{H} 1 \mathrm{~N} 1$ influenza vaccine and narcolepsy following 4 cases per 100000 vaccinated in Sweden and 9 cases per 100000 vaccinated in Finland. ${ }^{19} 20$ These cases provide a valuable lesson for the COVID-19 vaccine community that not all vaccine safety concerns can be anticipated; occasionally unexpected but serious conditions can arise, which require rapid assessment irrespective of biological plausibility. However, one fundamental safety concern for a new COVID-19 vaccine, which requires consideration, is the potential for disease enhancement (vaccinated individuals developing more severe forms of the disease than if had they not been vaccinated). ${ }^{21}$ This may not be a risk with all COVID-19 vaccines and more dependent on the nature of the vaccine, for example, DNA/RNA vaccine versus conventional vaccine. The best approach is to monitor for all adverse events but with some emphasis on certain adverse events, including those that have been previously reported with vaccines, potential and identified risks highlighted from premarketing development and any adverse events attributed to the specific vaccine vector. Furthermore, with the production of a COVID-19 vaccine likely to occur on a global scale, atypical patterns related to specific batches may indicate manufacturing issues; these must be promptly identified. ${ }^{22}$

\section{Sample size}

While premarketing studies detect frequent events, often they will not achieve the sample size to detect rare adverse events ${ }^{17}$; this will be less likely with the smaller premarketing clinical trials in expedited COVID-19 vaccines development programmes. To test for vaccine efficacy, there is a need for risk of infection in the community. The recent lowering of viral transmission rates in the community, a measure of successful public health measures, may lead to difficulties in demonstrating true vaccine efficacy in the cohort sizes of clinical trials. Some trials for COVID-19 vaccines are being conducted in countries with high prevalence of COVID-19 infection. Nevertheless, vaccine monitoring in the postmarketing phase is required with much larger sample sizes, possibly several hundred thousand vaccinees or more. In addition, consideration should be given to the different types of vaccine that may be marketed with different dosing regimens. Sufficient sample sizes for each vaccine type and within subgroups of interest will be of vital importance to ensure that rare or very rare adverse events can be detected and to provide confidence in measures of effectiveness.

However, there are specific challenges relating to sample sizes of postmarketing studies that must not be overlooked. Achieving such large sample sizes would require first sufficient vaccination uptake in the population, for which there is some expected hesitancy. Secondly, for those individuals who have received the vaccine, high enrolment rates to postmarketing surveillance will be necessary. Achieving widespread recruitment for studies conducted in the UK will require collaboration with other institutions and partnership with the National Institute for Health Research (NIHR) Clinical Research Networks; an existing network of contacts and experience in conducting vaccine surveillance studies with the NIHR can ensure rapid implementation of a vaccine study across the UK, with minimal lead time. In addition, other recruitment approaches may need to be considered, such as widespread study advertisement in a broad range of media. For a COVID-19 vaccine active surveillance study, given the intense public interest, a very high participation/response rate would be expected.

\section{Real-time reporting}

If supplies are available, it is likely that an emergency plan will allow vaccination with a COVID-19 vaccine to be performed within weeks or months. To ensure widespread uptake, it is essential that public confidence in the vaccine is high. To support this real-time collection of data will be necessary. From a public health perspective, initial regular weekly reports to relevant stakeholders notifying on the detection of safety or lack of efficacy signals can provide a platform for prompt regulatory and public health actions and compensate for the relatively reduced efficacy and safety information at the time of vaccine launch. Such rapid reporting may also improve public confidence in the vaccination programme, addressing concerns about safety and effectiveness and encouraging uptake of the vaccine. Transparency in research is important to improve public trust.

\section{Monitoring uptake of vaccination programmes}

Global fear of COVID-19 will likely be supplemented by apprehension regarding vaccination among populations. A recent poll in the USA found that only $49 \%$ of Americans planned to receive a COVID-19 vaccine when available. ${ }^{23}$ Differences in willingness to be vaccinated has also been observed across Europe. An online survey in seven European countries revealed that Germany and France had the largest proportions (10\%) of the population opposed to COVID-19 vaccination; France also had the largest proportion $(28 \%)$ who were unsure about receiving a vaccination. ${ }^{24}$ While findings for the UK appeared more promising (approximately 80\% willing to receive vaccination), there is still a degree of uncertainty. This is further supported by a UK poll conducted by the Royal Society of Public Health that revealed that one in five individuals would either not get vaccinated or were not sure. ${ }^{25}$ Thus, it is important not to overlook the overall performance of vaccine immunisation programmes. Postauthorisation studies for COVID-19 vaccines will need to monitor vaccine utilisation to understand the demographic characteristics of those who are vaccinated and people who did not receive the vaccine. The efforts of fast-tracking a COVID-19 vaccine through premarketing stages must be met with equal efforts of promoting a high-enough vaccination rate to achieve vaccine-induced herd immunity. Key to the success of the vaccination programme is the confidence of the public in the vaccine's effectiveness and safety.

\section{Single vaccine studies versus head-to-head comparative studies}

With multiple vaccines in development with different modes of action and the anticipated simultaneous demands for vaccines worldwide, it is likely that more than one vaccine will be available for COVID-19. This may provide an opportunity to perform 


\section{Box 2 Evaluating more than one COVID-19 vaccine}

- Head-to-head vaccine comparisons may be useful when an unvaccinated control group is unavailable or considered inappropriate, but caution is still required in active comparator selection.

- Different vaccines may have different biological modalities. For example, one of the most advanced is an RNA vaccine developed by Moderna Therapeutics, but this uses an unproven platform. ${ }^{27}$ Other potential COVID-19 vaccines are based on vaccine technologies that are already established for use in vaccines against other viral diseases; an example is the ChAdOx1/AZD1222 vaccine developed by the University of Oxford, which uses an extensively studied chimpanzee adenovirus vector.

- Some potential COVID-19 vaccines use conventional vaccine technology, based on viral protein administered with an adjuvant to trigger a response by the innate immune system. ${ }^{28}$

- There have been safety concerns surrounding adjuvants with previous vaccines ${ }^{29}$; whether this is a potential for new COVID-19 vaccines also needs to be monitored.

- Should two vaccines be included in one study, there needs to be diligence in identifying the specific vaccine received by vaccinees. This also applies if two separate studies are designed, one for each vaccine.

- Furthermore, success of a postmarketing study for an efficacious vaccine is dependent on wellcoordinated production of the vaccine and robust supply chains. ${ }^{30}$ Providing a vaccine nationally and internationally presents with logistical challenges and financial costs; there have been concerns that supplies will struggle to meet demands in the immediate term. However, vaccine providers have come forward with plans for expanding production.

head-to-head comparative studies comparing the effectiveness and safety of one particular vaccine to another (box 2).

\section{Conclusions}

The approach to vaccine benefit and risk evaluation is a continuum throughout the process of vaccine development and life cycle of the vaccine from premarketing to postmarketing. For COVID-19, advancing vaccines quickly through the premarketing schedules merits even more rigorous postmarketing surveillance. This can be achieved through carefully designed postmarketing safety studies. Ideally, a COVID-19 vaccine postmarketing study should allow for active surveillance with timely collection of granular data directly from vaccinees and HCPs, evaluation across different population subgroups and provision of real-time reporting. With mass vaccination coverage anticipated, findings from immediate postmarketing data on COVID-19 vaccines can guide regulatory decisions and public health practice to maintain a positive benefit-risk balance.

Contributors This article is important because postmarketing data are essential to provide real-world evidence on the use, effectiveness and safety of COVID-19 vaccines. Advancing vaccines quickly through the premarketing schedules merits even more rigorous postmarketing surveillance. All authors are all pharmacoepidemiologists and have worked in the field of drug safety for many years. They have extensive experience monitoring the safety of medicines and vaccines in the postmarketing phase in the UK and used previous studies as reference material for this paper. SD wrote the first draft of the manuscript. VO, EL and SAWS assisted with the concept, study design and writing the manuscript. All authors reviewed, contributed to revisions and approved the manuscript and accept full responsibility for its overall content. SAWS is the guarantor of this article.

Funding The authors have not declared a specific grant for this research from any funding agency in the public, commercial or not-for-profit sectors.

Competing interests None declared.

Patient consent for publication Not required.

Provenance and peer review Not commissioned; externally peer reviewed.

Open access This is an open access article distributed in accordance with the Creative Commons Attribution Non Commercial (CC BY-NC 4.0) license, which permits others to distribute, remix, adapt, build upon this work non-commercially, and license their derivative works on different terms, provided the original work is properly cited, appropriate credit is given, any changes made indicated, and the use is non-commercial. See: http://creativecommons.org/licenses/by-nc/4.0/.

\section{ORCID iDs}

Sandeep Dhanda http://orcid.org/0000-0002-4831-2535

Vicki Osborne http://orcid.org/0000-0002-3669-6084

Elizabeth Lynn http://orcid.org/0000-0002-3905-1046

Saad Shakir http://orcid.org/0000-0002-4084-297X

\section{References}

1 Pronker ES, Weenen TC, Commandeur H, et al. Risk in vaccine research and development quantified. PLoS One 2013;8:e57755-e55.

2 Mahase E. Covid-19: what do we know so far about a vaccine? BMJ 2020;369:m 1679 .

3 World Health Organization. Who guidelines on clinical evaluation of vaccines. regulatory expectations. Annex 1, 2001. Available: https://www. who.int/biologicals/publications/clinical_guidelines_ecbs_2001.pdf?ua=1

4 Lurie N, Saville M, Hatchett R, et al. Developing Covid-19 vaccines at pandemic speed. N Engl J Med 2020;382:1969-73.

5 Ferguson NM, Laydon D, Nedjati-Gilani G. Impact of non-pharmaceutical interventions (NPIS) to reduce COVID-19 mortality and healthcare demand. Imperial College London, 2020.

6 Ioannidis J. The infection fatality rate of COVID-19 inferred from seroprevalence data. medRxiv 2020.

7 Wang F, Kream RM, Stefano GB. An evidence based perspective on mRNASARS-CoV-2 vaccine development. Med Sci Monit 2020;26:e924700-e00.

8 World Health Organization. Draft landscape of COVID-19 candidate vaccines - 3rd September 2020. Available: https://www.who.int/whodocuments-detail/draft-landscape-of-covid-19-candidate-vaccines [Accessed 7 Sep 2020].

9 A phase II clinical trial to evaluate the recombinant vaccine for COVID-19 (adenovirus vector) (CTII-nCoV), 2020. Available: https://clinicaltrials.gov/ ct2/show/NCT04341389

10 Koch T, Dahlke C, Fathi A, et al. Safety and immunogenicity of a modified vaccinia virus Ankara vector vaccine candidate for middle East respiratory syndrome: an open-label, phase 1 trial. Lancet Infect Dis 2020;20:827-38.

11 Plotkin SA, Orenstein WA, Offit PA. Vaccines. 5th ed. Philadelphia: Saunders, 2008. 
12 European Medicines Agency. Guideline on Good Pharmacovigilance Practice (GVP) Module V - Risk Management System (Rev 2). Available: https://www.ema.europa.eu/en/documents/scientific-guideline/guidelinegood-pharmacovigilance-practices-module-v-risk-management-systemsrev-2_en.pdf [Accessed 7 Sep 2020].

13 World Health Organization. Design of vaccine efficacy trials to be used during public health emergencies - points of considerations and key principles. Available: http://www 10.who.int/blueprint/what/normsstandards/AP1_guidelines_Online_Consultation.pdf

14 McNaughton R, Lynn E, Osborne V, et al. Safety of intranasal quadrivalent live attenuated influenza vaccine (QLAIV) in children and adolescents: a pilot prospective cohort study in England. Drug Saf 2016;39:323-33.

15 Mackenzie IS, MacDonald TM, Shakir S, et al. Influenza H1N1 (swine flu) vaccination: a safety surveillance feasibility study using self-reporting of serious adverse events and pregnancy outcomes. Br J Clin Pharmacol 2012;73:801-11.

16 European network of centres for pharmacoepidemiology and pharmacovigilance. ENCePP guide on methodological standards in pharmacoepidemiology, 2020. Available: http://www.encepp.eu/standards_ and_guidances/methodologicalGuide10_2.shtml

17 Sturkenboom M, Bahri P, Chiucchiuini A, et al. Why we need more collaboration in Europe to enhance post-marketing surveillance of vaccines. Vaccine 2019. doi:10.1016/j.vaccine.2019.07.081. [Epub ahead of print: 31 Oct 2019].

18 Schonberger LB, Bregman DJ, Sullivan-Bolyai JZ, et al. Guillain-Barre syndrome following vaccination in the National Influenza Immunization Program, United States, 1976--1977. Am J Epidemiol 1979;110:105-23.

19 Persson I, Granath F, Askling J, et al. Risks of neurological and immunerelated diseases, including narcolepsy, after vaccination with Pandemrix: a population- and registry-based cohort study with over 2 years of followup. J Intern Med 2014;275:172-90.

20 Nohynek H, Jokinen J, Partinen M, et al. AS03 adjuvanted AH1N1 vaccine associated with an abrupt increase in the incidence of childhood narcolepsy in Finland. PLoS One 2012;7:e33536-e36.
21 de Alwis R, Chen S, Gan ES, et al. Impact of immune enhancement on Covid-19 polyclonal hyperimmune globulin therapy and vaccine development. EBioMedicine 2020;55:102768.

22 European Medicines Agency. Guideline on good pharmacovigilance practice. product or population specific considerations I: vaccines for prophylaxis against infectious diseases, 2013. Available: https:// www.ema.europa.eu/en/documents/scientific-guideline/guidelinegood-pharmacovigilance-practices-gvp-product-population-specificconsiderations-i-vaccines_en.pdf

23 Mello MM, Silverman RD, Omer SB. Ensuring uptake of vaccines against SARS-CoV-2. N Engl J Med 2020. doi:10.1056/NEJMp2020926. [Epub ahead of print: 26 Jun 2020].

24 Neumann-Böhme S, Varghese NE, Sabat I, et al. Once we have it, will we use it? a European survey on willingness to be vaccinated against COVID-19. Eur J Health Econ 2020;21:977-82.

25 Royal Society of Public Health. One in five public unsure about getting coronavirus vaccine, 2020. Available: https://www.rsph.org.uk/about-us/ news/one-in-five-public-unsure-about-getting-coronavirus-vaccine-ifavailable.html [Accessed $31 \mathrm{Jul}$ 2020].

26 European Medicines Agency. COVID-19: how EMA fast-tracks development support and approval of medicines and vaccines, 2020. Available: https://www.ema.europa.eu/en/news/covid-19-how-ema-fasttracks-development-support-approval-medicines-vaccines

27 Diamond MS, Pierson TC. The challenges of vaccine development against a new virus during a pandemic. Cell Host Microbe 2020;27:699-703.

28 Zhang J, Zeng $\mathrm{H}$, Gu J, et al. Progress and prospects on vaccine development against SARS-CoV-2. Vaccines 2020;8:153.

29 Petrovsky N. Comparative safety of vaccine adjuvants: a summary of current evidence and future needs. Drug Saf 2015;38:1059-74.

30 Newton PN, Bond KC, et al, 53 signatories from 20 countries. COVID-19 and risks to the supply and quality of tests, drugs, and vaccines. Lancet Glob Health 2020;8:e754-5. 